\title{
Lunching for Relaxation or Cognitive Control? After-Effects of Social and Solitary Meals
}

\author{
Birgit Stürmer ', Guang Ouyang 2,3, Marina Palazova ', Annekathrin Schacht ${ }^{4}$, \\ Manuel Martín-Loeches ${ }^{5}$, Philip Rausch ${ }^{6}$, and Werner Sommer ${ }^{6}$ \\ 'International Psychoanalytic University Berlin, Stromstraße 1, 10555 Berlin, Germany \\ ${ }^{2}$ Department of Physics, Hong Kong Baptist University, Baptist University Rd., Kowloon Tong, Hong Kong \\ ${ }^{3}$ Centre for Nonlinear Studies and The Beijing-Hong Kong-Singapore Joint Centre for Nonlinear and Complex Systems, \\ Hong Kong Baptist University, Baptist University Rd., Kowloon Tong, Hong Kong \\ ${ }^{4}$ University of Göttingen, Wilhelmsplatz 1, 37073 Göttingen, Germany \\ ${ }^{5}$ Universidad Complutense de Madrid, Avda. de Séneca, 2, Ciudad Universitaria, 28040 Madrid, Spain \\ ${ }^{6}$ Humboldt-Universität zu Berlin, Unter den Linden 6, 10099 Berlin, Germany
}

ABSTRACT

\section{KEYWORDS}

meal, positive mood,

cognitive control

Meals, especially when taken in company, may affect the diner's mood. In line with findings that mood may alter cognitive control, a previous study by the authors found that after solitary meals, the Simon effect was diminished as compared to a premeal condition, whereas a social meal did not reduce the Simon effect. Here, we investigated whether this finding generalizes across different demands in cognitive control and, therefore, applied a flanker task. Obtained questionnaire data indicated differential effects in mood and relaxation of a social as compared to a solitary meal. Replicating our previous findings, the flanker compatibility effect decreased after a solitary meal but increased after a social meal. The present results support our previous findings with new evidence that a meal taken in a social context attenuates subsequent cognitive control processes compared with a solitary meal.

\section{INTRODUCTION}

Meals are indispensable elements of human life, from providing basic nutrients up to the elaborate culinary experiences staged in top gastronomy. For the experience of the meal, it seems to become increasingly clear that not only the food itself is important but also the environment in which the meal is consumed (Meiselman, 2008; Spence \& Piqueras-Fiszman, 2014). Although there are strong popular believes on the effects of commensality, most famously expressed in the story Babette's Feast by Karen Blixen (2011), surprisingly little formal research has been done about the psychological consequences of the social setting of meals.

Recently, we reported that cognitive control was diminished after a meal when taken in company of another person and in a restaurant, relative to a solitary meal in a plain environment (Sommer, Stürmer, Shmuilovich, Martin-Loeches, \& Schacht, 2013). In order to induce cognitive conflicts, we had used the Simon task, where spatially arranged choice responses have to be performed on the basis of nonspatial stimulus features. Although task irrelevant, stimuli are presented at spatial positions overlapping with the responding hand placements. The Simon effect refers to the finding that responses are slower and more error-prone in incompatible conditions when stimulus and

Corresponding author: Prof. Dr. Birgit Stürmer, International Psychoanalytic University, Stromstr. 1, 10555 Berlin, Germany. Phone: ++49 30300117725. Email: birgit.stuermer@ipu-berlin.de 
response location mismatch as compared to compatible conditions where they match. Dual route models (De Jong, Liang, \& Lauber, 1994; Kornblum, Hasbroucq, \& Osman, 1990) account for the Simon effect by suggesting that instruction-induced response selection is accomplished via an indirect route. In parallel, the spatial dimension is processed via a second-direct-route that primes the spatial response according to stimulus location. In incompatible trials, a cognitive conflict is induced because the spatial response position primed by the direct route differs from the response selected by the indirect route. The Simon effect is strongly reduced when cognitive control is applied to direct route processing and prevents response priming (Stürmer \& Leuthold, 2003), and it increases when cognitive control is alleviated (Stürmer, Redlich, Irlbacher, \& Brandt, 2007).

In our previous study, the Simon effect decreased after the solitary meal situation of the control group but remained unaltered in the social meal group, compared to a baseline session that both groups completed without any food intake a week before. In addition, the errorrelated negativity as a correlate of error monitoring in the event-related brain potential (ERP) increased in the control group after the plain meal compared to the baseline session. In contrast, the error-related positivity as an indicator of error awareness in the ERP was diminished after the social meal compared to the baseline session. Moreover, ERPs to threatening faces were enlarged after the social meal during a gender decision task, calling for choice responses to emotional face stimuli. Taken together, these findings are in line with the idea that a social meal has a positive, relaxing effect on mood and reduces cognitive control.

Such findings are important from a health perspective because they indicate that a social meal break may have a stronger stress reducing effect than a solitary brown bag meal. However, the results are also important from a basic research perspective concerning the consequences of affective states on cognitive control (Clore \& Huntsinger, 2007; Forgas, 2008). From a basic point of view, top-down control of behavior deals with two mutual exclusive requirements, that is, behavioral stability versus flexibility (see Goschke \& Bolte, 2014; Hommel, 2015 , for recent reviews). On the one hand, current action goals have to be shielded against concurrent goals to guarantee goal achievement for the sake of behavioral stability. On the other hand, there is the need to flexibly shift goals according to new external or internal demands. Goal shielding is often accompanied by narrowly focused attention, allowing for the suppression of distracting information, whereas flexibility requires monitoring the environment and is therefore supported by more distributed attention. Cognitive-affective interactions in the context of the requirements for stability versus flexibility have been widely discussed. For example, Dreisbach and Goschke (2004) showed that positive affect relaxes cognitive control by increasing cognitive flexibility and, hence, task-irrelevant information is more distracting. In a similar vein, Zwosta, Hommel, Goschke, and Fischer (2013) showed that negative mood results in less cross-talk in a dual task situation and, hence, enhances goal shielding. These findings support the general assumption that positive mood widens the attentional focus and induces flexibility, whereas negative mood focusses attention and facilitates shielding.
With the present study, we aimed to extend previous behavioral findings to a different cognitive conflict task, that is, we used a flanker rather than a Simon task. Mood-induced modulations of the flanker effect have been previously reported. For example, Rowe, Hirsh, and Anderson (2007) observed an enlarged flanker effect when participants reported to be happy. Although response conflicts have been demonstrated in both the flanker and the Simon task, conflict control mechanisms differ. In contrast to the Simon task, where conflict control is applied to response preparation processes (Kornblum et al., 1990), response conflicts triggered in the flanker task are controlled by enhancing the focus of attention on the task-relevant stimulus (Gratton, Coles, \& Donchin, 1992; Scerif, Worden, Davidson, Seiger, \& Casey, 2006). Flanker conflicts are usually stronger than Simon conflicts, therefore, enabling a larger range for modulation by mood. Hence, we expected the flanker conflict to increase after a social meal as compared to a baseline session without food intake.

As in our previous study, we tested only female participants because women have been shown to be more sensitive to mood inductions (Federmeier, Kirson, Moreno, \& Kutas, 2001), which might be at the bottom of commensality effects on cognitive control. In addition to the flanker task, we applied a prisoner's dilemma task and tasks on mental speed, semantic memory, and emotional facial expressions. These tasks were not related to cognitive conflict control. Furthermore, none of these tasks showed any effect according to our experimental manipulation. Therefore, and because tasks were always presented in a fixed order, we will not report the other tasks in the present paper.

\section{METHOD}

\section{Participants}

Forty women of diverse occupational background participated in the experiment; 20 each were assigned to the experimental (EG) and control group (CG), respectively, and matched pair-wise for age $\left(M_{\text {age }}=\right.$ 23.9 , for the range of 20 to 29 years, vs. $M_{\text {age }}=24.0$, for the range of 21 to 30 years) and body mass index (BMI; weight $[\mathrm{kg}] /(\text { height }[\mathrm{m}])^{2} ; M=$ 21.5 , for the range of 18.7 to 25.1 , vs. $M=21.7$, for the range of 18.5 to 24.9). The EG and CG were also matched according to Oldfield's (1971) handedness questionnaire; mean scores were 66 (range: -100 to 100) and 72 (range: -66 to 100), respectively. Due to an unusually high error rate in the flanker task both in the premeal (22\%) and postmeal session, one participant in the CG was excluded from further analyses. Her demographic data were already excluded from the above-mentioned values. None of the participants had any special dietary restrictions, for example, accepting only vegan food. Vegetarians were included in the CG only if the matched person from the EG also accepted vegetarian food. None of the participants reported a history of food allergies, psychiatric, or neurological disorders. To exclude current depressive states, the Beck Depression Inventory (BDI-II, Hautzinger, Keller, \& Kühner, 2006) was applied, using a cut-off score of 13 (range: 0 to 63).

Food and soft drinks were provided for free to the participants and their meal companions, the time invested in the pre- and postmeal ses- 
sion was reimbursed with $8 € /$ hr. All participants had given written, informed consent. The study was approved by the local ethics committee of the Department of Psychology of the Humboldt-Universität zu Berlin and conducted in accordance with the Declaration of Helsinki. Participants were informed in writing that the aim of the study was to assess the effects of a meal on some psychological functions.

\section{Questionnaires}

Mood states were assessed with the long form of the Multidimensional Mood State Questionnaire (MDBF; Steyer, Schwenkmezger, Notz, \& Eid, 1997). The MDBF consists of 24 mood adjectives, which are rated according to their fit with the current mood on 5-point Likert scales (from definitely not to very much). The answers yield scores on the scales bad to good mood (BG), sleepy to awake (SA), and restless to calm (RC; range of scores on all scales: 8 to 40 ).

A restaurant and meal questionnaire with Likert-scale items (ranged from 1, very good, to 6, insufficient) about the restaurant's quality and atmosphere, as well as the meal's tastiness, was administered to the EG. The CG rated meal tastiness and atmosphere of the office room on the same scales.

\section{Procedure}

The study consisted of three sessions: premeal, meal, and postmeal. The pre- and postmeal sessions were identical for both EG and CG and measured a range of psychological and psychophysiological variables; the crucial experimental factor-the social meal context-was manipulated by the meal session. Participants were asked to refrain from excessive consumption of alcoholic beverages on the evenings before testing and during testing days. Breakfast habits should not be altered, but breakfast should be taken at least four hours before the meal session.

Premeal sessions always started at 9 AM, for both the CG and the EG, with signing an informed consent followed by measuring the BMI and by answering the BDI-II, followed by a demographic and handedness questionnaire, and the MDBF. After the completion of two behavioral tasks (prisoner's dilemma, mental speed), EEG electrodes were applied, followed by three experimental tasks. The first task was the flanker task, followed by a task on semantic memory and emotional facial expressions. The data from the EEG recordings and the other tasks will not be reported here. Altogether, tasks during this session took around $40 \mathrm{~min}$.

In the flanker task, stimuli were upper-case letters arranged horizontally; a gray $\left(54 \mathrm{~cd} / \mathrm{m}^{2}\right)$ target letter (visual angle: $0.5^{\circ} \times 0.7^{\circ}$ ) was presented at center on a dark gray $\left(45 \mathrm{~cd} / \mathrm{m}^{2}\right)$ monitor and was flanked by four identical white $\left(66 \mathrm{~cd} / \mathrm{m}^{2}\right)$ letters (visual angles $\left.0.5^{\circ} \times 0.7^{\circ}\right)$, two on each side of the central target letter, yielding stimulus arrays of $2.9^{\circ}$ $\times 0.7^{\circ}$. The letters $H, N, V$, and $X$ were used as targets and flankers, randomized across response hands. The lower contrast for targets than for flankers aimed to increase flanker effects. Each trial started with the presentation of a gray fixation point for $500 \mathrm{~ms}$ followed by the flanker stimuli; $150 \mathrm{~ms}$ later, a target letter appeared for $100 \mathrm{~ms}$; then, all letters were replaced by the fixation point, remaining on the screen for $1.5 \mathrm{~s}$, until the next trial started. Participants responded to the target letters with the index fingers of the right and left hand placed on corresponding response keys. Two out of four target letters were assigned to each response hand, and four different combinations of letters were counterbalanced across participants. Compatible and incompatible trials, that is, where target and flanker letters were the same or different, respectively, were presented with equal probability (50\% each) and in random order. Response feedback was provided during 36 practice trials, followed by 480 test trials without feedback. After every 80 trials, a break was provided; the flanker task's duration was $20 \mathrm{~min}$.

Meal sessions took place approximately one week after the premeal session and started between $12 \mathrm{AM}$ and 1 PM for both the CG and EG. Meals were provided by two Italian restaurants within 10 min walking distance from the laboratory. Both restaurants offered a restricted menu to our participants, consisting of vegetarian and nonvegetarian pasta and pizza dishes and soft drinks. For the EG, the meal sessions took place in one of the restaurants during their normal operation times. Hence, other guests were present and background music played as usual. Meals and drinks could be freely chosen from the restricted menu; in every other respect, our participants were treated as all other guests. Importantly, participants should invite a companion of their choice for lunch, someone they would typically dine with, and were encouraged to spend one hour in the restaurant. Immediately following lunch and after the accompanying person had left the restaurant, participants filled in the MDBF and meal questionnaire. In case a participant did not finish her meal, she was asked to bring the left overs in a doggy bag to the laboratory, where they were weighed in order to adapt meal size in the matched partner of the CG.

Participants in the CG also walked to the restaurant and picked up their packaged and sealed meals. CG members received the same meal as their matched partner from the EG and hence had no free choice of meals. They took their meal to an office room close to the laboratory where they dined alone without entertainment or distraction, such as internet access, smartphone, or music. If the matched EG participant had not finished her meal, the experimenter reduced the meal of the CG participant accordingly. The CG participants had $20 \mathrm{~min}$ to finish their meals - which is usually ample time-after which they completed the MDBF and the meal questionnaire.

The postmeal session immediately followed the meal session on the same day and was identical with the premeal session, excluding questionnaires.

\section{Data Analysis}

Statistical analyses were conducted within the statistical computing environment $R$ (R Development Core Team, 2013). The behavioral data were analyzed by means of linear mixed effects models (LMMs; Baayen, Davidson, \& Bates, 2008) using the lmer function of the lme4 package (Bates, Maechler, \& Bolker, 2013). Prior to analysis of response accuracy and reaction times (RTs), missed trials as well as all trials with RTs $<100$ ms or $>1$ s were excluded. For the RT analyses, trials with incorrect responses were additionally excluded. Afterwards, RTs were log-transformed and trimmed separately for each data set, removing 
any trials with $\log$-RTs $>2.5 S D$ of the mean. The remaining singletrial $\log$ RTs entered the model as a dependent variable. In addition to an intercept effect, the LMMs comprised fixed effect terms for factors of Compatibility, Session, and Group, as well as all their interactions. The random effects were modeled by including a by-subject random intercept, as well as random slopes for the within-subject factors of Compatibility and Session and their interaction, resulting in a maximal by-subject random effects structure (Barr, Levy, Scheepers, \& Tily, 2013). To reduce collinearity, all variables were centered at their means before analysis. Note that $p$ values for fixed effects for Gaussian models fitted with the lmer function are not available as part of the default output. The $p$ values presented below were computed with the glht function of package multcomp (Hothorn, Bretz, \& Westfall, 2008); the significance of relevant predictors was also confirmed with likelihoodratio tests. The binary response accuracy data were analyzed with a logistic LMM using the same fixed and random effects specification as for the RT model. The $p$ values for this model are based upon the Wald $z$ score. As Jaeger (2008) points out, logistic LMMs are more adequate tools for the analysis of binary outcome data (such as response accuracy) than analyses of variance (ANOVAs). In order to allow direct comparison to previous studies, however, ANOVA results including the same factors as the LMM analysis are reported next to LMM results for RTs, together with the generalized eta squared $\left(\eta_{\mathrm{G}}{ }^{2}\right.$; Olejnik \& Algina, 2003) as a measure of effect size. RT estimates given are based on LMM estimates; indicated SEs and CIs for the RT and response accuracy analyses were obtained from the LMMs using the Effect function (package effects; Fox, 2003). For the response accuracy results, we report estimates of the error probabilities and associated SEs based on the logistic LMM. Post-hoc tests were carried out using paired $t$ tests for single comparisons and LMMs/ANOVAs where appropriate. As all comparisons were planned, we did not apply Bonferroni corrections for post hoc tests. To test for baseline differences between groups in the premeal session, we applied an ANOVA, including the factors of Group and Compatibility on RTs of the premeal session.

Questionnaire data were analyzed using mixed ANOVAs with Compatibility and Session as within-subject factors and Group as between-subjects factor.

\section{RESULTS}

\section{Questionnaires}

Pre- and postmeal scores of the MDBF questionnaire did not show any group or session differences. Similar to our previous study (Sommer et al., 2013), no interaction between group and session was observed in any of the three scales. Post hoc $t$ tests of the individual scales revealed a trend in the CG for a decrease of $\operatorname{mood}, t(38)=2.02, p=.058$, and an increase of restlessness, $t(38)=2.09, p=.05$.

While the tastiness of the meals was rated similarly in both groups $\left(M_{\mathrm{EG}}=1.9\right.$ vs. $\left.M_{\mathrm{CG}}=2.1 ; t<1.00\right)$, the atmosphere of the restaurants was rated significantly better than the lunch situation at the office $\left(M_{\mathrm{EG}}\right.$

\section{TABLE 1.}

Mean Values and SDs of the Three Scales in the Multidimensional Mood State Questionnaire (MDBF) per Group and Session

\begin{tabular}{ccccccc}
\hline & \multicolumn{2}{c}{ BG } & \multicolumn{2}{c}{ SA } & \multicolumn{2}{c}{ RC } \\
\cline { 2 - 7 } & EG & CG & EG & CG & EG & CG \\
\hline Pre- & 33.9 & 36.2 & 27.2 & 29.4 & 32.1 & 35.0 \\
meal & $(4.0)$ & $(2.9)$ & $(6.8)$ & $(7.2)$ & $(4.4)$ & $(3.4)$ \\
Post- & 33.8 & 34.1 & 26.5 & 28.1 & 32.3 & 33.0 \\
meal & $(4.8)$ & $(4.8)$ & $(5.9)$ & $(7.2)$ & $(4.9)$ & $(5.1)$ \\
\hline
\end{tabular}

Note. $\mathrm{BG}=$ bad to good mood scale; $\mathrm{SA}=$ sleepy to awake scale; $\mathrm{RC}=$ restless to calm scale; $\mathrm{EG}=$ experimental group; $\mathrm{CG}=$ control group.

$=1.6$ vs. $\left.M_{\mathrm{CG}}=3.0\right), t(38)=4.92, p<.05$. No significant differences were observed between the two restaurants (all $t \mathrm{~s}<1.6$ ).

\section{Performance}

In general, mean error rates in the flanker task were low, ranging between 4.1 and 5.1\%. The LMM results show an expected main effect of compatibility, $z=4.34, p=.000014$, (compatible: prob $_{\text {Error }}=0.031, S E=$ 0.0038 ; incompatible: prob $_{\text {Error }}=0.044, S E=0.0052$ ) and a main effect of session, $z=2.65, p=.008$, reflecting a practice effect from the premeal session ( prob $_{\text {Error }}=0.041, S E=0.0046$ ) to the postmeal session (prob $b_{\text {Error }}$ $=0.032, S E=0.0045)$. A similar main effect of session was seen for RTs $\left(M_{\text {premeal }}=505, S E=7.9\right.$, vs. $\left.M_{\text {postmeal }}=490 \mathrm{~ms}, S E=7.6\right)$, LMM: $t=2.75$, $p=.006$; ANOVA: $F(1,37)=7.37, p=.01, \eta_{\mathrm{G}}{ }^{2}=.024$. As expected, there was a large effect of flanker compatibility $\left(M_{\text {comp }}=471 \mathrm{~ms}, S E=7.6\right.$, vs. $\left.M_{\text {incomp }}=526 \mathrm{~ms}, S E=7.3\right)$; LMM: $t=13.89 ; p=2^{-16}$; ANOVA: $F(1,37)$ $=192.41, p=4^{-16}, \eta_{\mathrm{G}}{ }^{2}=.244$. Most importantly, there was a three-way interaction of compatibility, group, and session (see Figure 1), LMM: $t$ $=2.54, p=.011$; ANOVA: $F(1,37)=6.61, p=.014, \eta_{\mathrm{G}}{ }^{2}=.002$. The compatibility effect in the CG decreased from $M_{\text {difference }}=61 \mathrm{~ms}$ to $52 \mathrm{~ms}$, LMM: $t=1.82, p=.035$ (one-tailed); ANOVA: $F(1,18)=3.36, p=.042$ (one-tailed), $\eta_{\mathrm{G}}{ }^{2}=.002$, whereas in the EG, it increased from $51 \mathrm{~ms}$ to 56 ms, LMM: $t=1.81, p=.03$ (one-tailed); ANOVA: $F(1,19)=3.28, p=$ .043 (one-tailed), $\eta_{\mathrm{G}}{ }^{2}=.002$. As indicated in Figure 1, the compatibility effect in the premeal session is descriptively larger in the CG compared to the EG. An ANOVA on premeal RTs, however, showed neither a significant interaction of group and compatibility, $F(1,37)=1.94, p=$ $0.172, \eta_{\mathrm{G}}{ }^{2}=.004$, nor a significant main effect of group, $F<1.00$. These results rule out baseline differences between groups as a cause for the three-way interaction above.

\section{DISCUSSION}

With the present study, we aimed at replicating and extending our previous findings that meal situations affect cognitive control, by using a similar design as realized in our previous study (Sommer et al., 2013) but in a different cognitive conflict task. The tastiness of the meals was rated as good, regardless of where it was consumed. As to be expected, the atmosphere in the restaurant was judged as better than that of the 

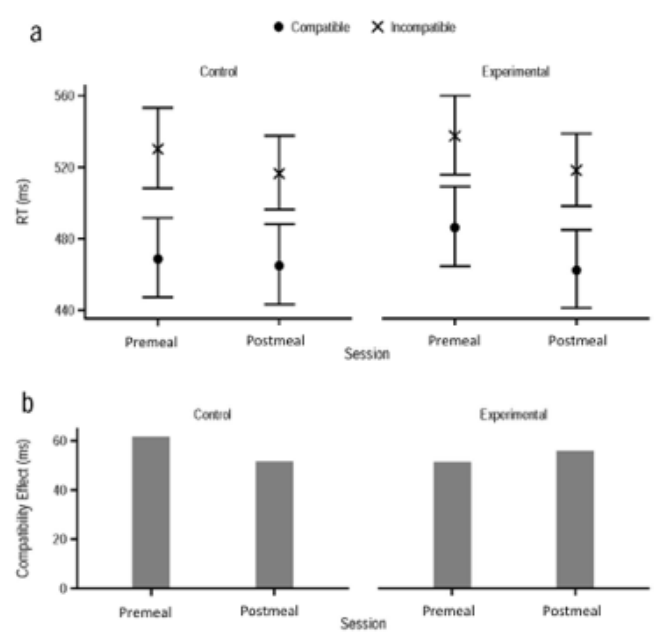

\section{FIGURE 1.}

Reaction times (RTs) in the flanker task. Panel A: Cell-mean estimates with $95 \%$ Cls. Panel B: Compatibility effect. All displayed quantities are based on linear mixed effect model (LMM) estimates.

office room. However, the office meal atmosphere was still rated as satisfactory, whereas the restaurant was rated as good.

Data from mood questionnaires indicated that the CG was more restless in the postmeal relative to the premeal session and-at least with a strong trend-reported less positive mood in the postmeal session. In contrast, subjectively reported mood was stable in the EG across the two sessions.

The flanker task showed the expected effects of incompatibility and practice across sessions. Importantly, it fully replicated our previous findings with a different conflict task for the CG, where the compatibility effect decreased after the solitary meal as compared to the premeal session. As expected, the flanker conflict increased in the EG after the social meal compared to the premeal session. Sommer et al. (2013) have found a stable Simon effect in the EG across sessions. The missing decrease of the Simon effect after the social meal was taken as evidence of relaxed cognitive control, admittedly a somewhat indirect conclusion. The increased flanker effect after the social meal in the present study validates our assumption of relaxed cognitive control after a social meal. It is, however, an open question whether positive mood triggers relaxation of control or whether, instead, negative mood strengthens control (Botvinick, 2007; Dreisbach \& Fischer, 2015). Concerning this question, our present data are not decisive. Mood was stable across both sessions in the EG whereas there was a-nonsignificant-trend towards a decrease in the CG. Descriptively, on the BG scale of the MDBF (see Table 1), the mood in the CG started off somewhat better than in the EG in the premeal session but decreased to the same level as in the EG in the postmeal session. Moreover, ratings of the meal situations indicated that even the CG rated the lunch situation as satisfactory.
Performance in the flanker task showed a decrease of RTs from the premeal to the postmeal session. We ascribe this general reduction of RTs from the first to the second session to practice. Overall decreases of RTs with practice have been observed also in compatibility tasks (Dutta \& Proctor, 1992; Proctor \& Lu, 1999). Moreover, the compatibility effect also decreased with practice, along with overall shorter RTs. In the present study, for the CG, we observed the expected smaller flanker effect with overall shorter RTs in the postmeal session.

An alternative account for the reduced flanker effect after a solitary meal is related to the idea that negative mood or the experience of aversive signals triggers goal shielding processes by increasing cognitive control. The attentional focus narrows onto the target stimulus in the flanker task, thus reducing flanker interference. Although ratings on the BG scale of the MDBF questionnaire slightly decreased in the CG, these ratings were still within the range of good $\operatorname{mood}(M=34.1$; scale range: 8 to 40 ). As questionnaire data did not show any signs of negative mood or aversiveness, we prefer to attribute the reduced flanker effect in the postmeal session of the CG to practice.

For the EG, however, the flanker effect increased with shorter RTs in the postmeal session compared to the premeal session. This is a rather unusual finding that we take as indication of relaxed cognitive control triggered by the social meal experience. In all, this is a remarkable replication with a very similar finding across different labs, restaurants, and conflict-inducing tasks. Therefore, we may conclude that the differences in the meal situations reliably affect control processes in subsequent cognitive conflict situations.

We kept the time of day when the measurement was applied constant between groups. Both the CG and the EG had their premeal session at $9 \mathrm{AM}$, and the second sessions after one week started at noon for both groups. Testing time, therefore, could not account for differences between groups. Meal situations between the EG and CG, however, differed in more than one relevant variable: First of all, the participants in the EG were accompanied by a friend, therefore, they took their meal in a social context, whereas participants in the CG ate alone. Moreover, the EG was in a restaurant with a likely stimulating atmosphere, other guests, background music, and so on, whereas the CG dined in an office without any entertainment. Thirdly, participants in the EG could choose their meal from a menu, whereas the CG had no choice. On the basis of present findings we cannot conclude which of these variables contributed to the observed differences between the EG and the CG. We believe that the observed group differences are not due to a single variable but may be caused by the combination of restaurant ambience, a long break, and the company of a friend.

In conclusion, we could demonstrate attenuation of cognitive control after a meal taken in a social context. This replication and extension of our previous findings emphasizes the role of meal context not only during the meal but also for behavioral regulation afterwards. The decrease in cognitive control of a social relative to a solitary meal (without work or media use) allows for an informed decision whether to emphasize on stress management or cognitive control by choosing the context for lunch. 


\section{REFERENCES}

Baayen, R. H., Davidson, D. J., \& Bates, D. M. (2008). Mixed-effects modeling with crossed random effects for subjects and items. Journal of Memory and Language, 59, 390-412. doi: 10.1016/j. jml.2007.12.005

Barr, D. J., Levy, R., Scheepers, C., \& Tily, H. J. (2013). Random effects structure for confirmatory hypothesis testing: Keep it maximal. Journal of Memory and Language, 68, 255-278. doi: 10.1016/j.jml.2012.11.001

Bates, D. M., Maechler, M., \& Bolker, B. (2013). Ime4: Linear mixedeffects models using S4 classes. Retrieved from http://CRAN.Rproject.org/package=Ime4 (R package version: 0.999999-2).

Blixen, K. (2011). Babette's Feast. New York, NY: Penguin Group.

Botvinick, M. (2007). Conflict monitoring and decision making: reconciling two perspectives on anterior cingulate function. Cognitive, Affective and Behavioral Neuroscience, 7, 356-366.

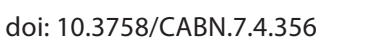

Clore, G. L., \& Huntsinger, J. R. (2007). Cognitive-emotional interactions: How emotions inform judgment and regulate thought. Trends in Cognitive Sciences, 11, 393-399. doi: 10.1016/j.tics.2007.08.005

De Jong, R., Liang, C. C., \& Lauber, E. (1994). Conditional and unconditional automaticity: A dual-process model of effects of spatial stimulus-response correspondence. Journal of Experimental Psychology: Human Perception and Performance,

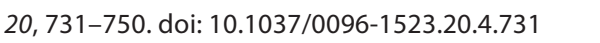

Dreisbach, G., \& Fischer, R. (2015). Conflicts as aversive signals for control adaptation. Current Directions in Psychological Science, 24, 255-260. doi: 10.1177/0963721415569569

Dreisbach, G., \& Goschke, T. (2004). How positive affect modulates cognitive control: Reduced perseveration at the cost of increased distractibility. Journal of Experimental Psychology: Learning, Memory, and Cognition, 30, 343-353. doi:

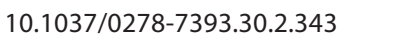

Dutta, A., \& Proctor, R. W. (1992). Persistence of stimulus-response compatibility effects with extended practice. Journal of Experimental Psychology: Learning, Memory, and Cognition, 18, 801-809. doi: 10.1037/0278-7393.18.4.801 |wWW|

Federmeier, K. D., Kirson, D. A., Moreno, E. M., \& Kutas, M. (2001). Effects of transient, mild mood states on semantic memory organization and use: An event-related potential investigation in humans. Neuroscience Letters, 305, 149-152. doi: 10.1016/ S0304-3940(01)01843-2 WWW

Forgas, J. P. (2008). Affect and cognition. Perspectives on Psychological Science, 3, 94-101. doi: 10.1111/j.1745-6916

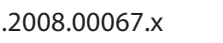

Fox, J. (2003). Effect displays in $\mathrm{R}$ for generalised linear models. Journal of Statistical Software, 8, 1-27. doi: 10.18637/jss.v008. i15

Goschke, T., \& Bolte, A. (2014). Emotional modulation of control dilemmas: The role of positive affect, reward, and dopamine in cognitive stability and flexibility. Neuropsychologia, 62, 403-423. doi: 10.1016/j.neuropsychologia.2014.07.015 WWW
Gratton, G., Coles, M. G. H., \& Donchin, E. (1992). Optimizing the use of information: Strategic control of activation of responses. Journal of Experimental Psychology: General, 121, 480-506. doi: 10.1037/0096-3445.121.4.480 WWW

Hautzinger, M., Keller, F., \& Kühner, C. (2006). Beck DepressionsInventar (BDI-II). Revision. [Beck Depression Inventory (BDIII). Revision]. Frankfurt am Main, Germany: Harcourt Test Services.

Hommel, B. (2015). Between persistence and flexibility: The yin and yang of action control. In A. J. Elliot (Ed.), Advances in motivation science (Vol. 2, pp. 33-67). Amsterdam, The Netherlands: Elsevier.

Hothorn, T., Bretz, F., \& Westfall, P. (2008). Simultaneous inference in general parametric models. Biometrical Journal, 50, 346-363.

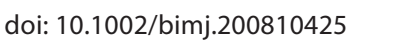

Jaeger, T. F. (2008). Categorical data analysis: Away from ANOVAs (transformation or not) and towards logit mixed models. Journal of Memory and Language, 59, 434-446. doi: 10.1016/j. jml.2007.11.007 $\underline{\underline{w W}}$

Kornblum, S., Hasbroucq, T., \& Osman, A. (1990). Dimensional overlap: Cognitive basis for stimulus-response compatibility-a model and taxonomy. Psychological Review, 97, 253-270. doi: 10.1037/0033-295X.97.2.253 $\underline{\underline{W W W}}$

Meiselman, H. L. (2008). Experiencing products within a physical and social context. In H. N. J. Schifferstein \& P. Hekkert (Eds.), Product experience (pp. 559-580). Oxford, England: Elsevier.

Oldfield, R. C. (1971). The assessment and analysis of handedness: The Edinburgh inventory. Neuropsychologia, 9, 97-113. doi: 10.1016/0028-3932(71)90067-4 |WWW

Olejnik, S., \& Algina, J. (2003). Generalized eta and omega squared statistics: Measures of effect size for some common research designs. Psychological Methods, 8, 434-447. doi: 10.1037/1082989X.8.4.434

Proctor, R. W., \& Lu, C.-H. (1999). Processing irrelevant location information: Practice and transfer effects in choice-reaction tasks. Memory \& Cognition, 27, 63-77. doi: 10.3758/BF03201214 WWW

R Development Core Team. (2013). R: A language and environment for statistical computing. Vienna, Austria. Retrieved from http://www.R-project.org/

Rowe, G., Hirsh, J. B., \& Anderson, A. K. (2007). Positive affect increases the breadth of attentional selection. Proceedings of the National Academy of Sciences United States of America, 104, 383-388. doi: 10.1073/pnas.0605198104 WWW

Scerif, G., Worden, M. S., Davidson, M., Seiger, L., \& Casey, B. J. (2006). Context modulates early stimulus processing when resolving stimulus-response conflict. Journal of Cognitive Neuroscience, 18, 781-792. doi: 10.1162/jocn.2006.18.5.781 WWW

Sommer, W., Stürmer, B., Shmuilovich, O., Martin-Loeches, M., \& Schacht, A. (2013). How about lunch? Consequences of the meal context on cognition and emotion. PLOS ONE, 8:e70314.

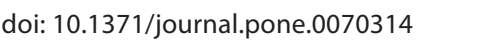


Spence, C., \& Piqueras-Fiszman, B. (2014). The perfect meal: The multisensory science of food and dining. Chichester, England: Wiley.

Steyer, R., Schwenkmezger, P., Notz, P., \& Eid, M. (1997). Der Mehrdimensionale Befindlichkeitsfragebogen (MDBF). Göttingen, Germany: Hogrefe.

Stürmer, B., \& Leuthold, H. (2003). Control over response priming in visuomotor processing: A lateralized event-related potential study. Experimental Brain Research, 153, 35-44. doi: 10.1007/ s00221-003-1579-1 [WW]
Stürmer, B., Redlich, M., Irlbacher, K., \& Brandt, S. A. (2007). Executive control over response priming and conflict: $A$ transcranial magnetic stimulation study. Experimental Brain Research, 183, 329-339. doi: 10.1007/s00221-007-1053-6 [wWw] Zwosta, K., Hommel, B., Goschke, T., \& Fischer, R. (2013). Mood states determine the degree of task shielding in dual-task performance. Cognition and Emotion, 27, 1142-1152. doi: 10.1080/02699931.2013.772047 WwW

RECEIVED 23.03.2017| ACCEPTED 28.11.2017 